\title{
Clinical Reasoning: A man with rapidly progressive weakness and respiratory failure
}

Denise Xu, BA, Kathleen Murphy, MD, Ramani Balu, MD, and Jon Rosenberg, MD

Correspondence

Neurology ${ }^{\circledR}$ 2018;91:e686-e691. doi:10.1212/WNL.0000000000006003

Dr. Rosenberg

jon.rosenberg@

uphs.upenn.edu

\section{Section 1}

A 70-year-old man presented in late summer with rapidly progressive weakness and respiratory failure. He was in his usual state of health until 1 week prior to presentation, when he developed malaise, headache, and fevers, followed by right leg weakness, prompting admission to a local hospital. Over the next 3 days, his weakness progressed to involve his right arm, then his extraocular, facial, and bulbar muscles, followed by his left arm and leg. On the fourth day of hospitalization ( 2 weeks after the onset of constitutional symptoms), he was intubated due to an inability to clear oral secretions and was transferred to our institution.

Upon arrival, the patient had a temperature of $102.3^{\circ}$, intact mental status, normal pupillary responses, restricted horizontal and vertical gaze, downbeating nystagmus on downward gaze, weakness of his bilateral upper and lower facial muscles, and reduced muscle tone. He had 0/5 strength in all extremities, save trace left finger movement, intact sensation without a sensory level, trace reflexes, and absent Babinski signs.

The patient has type 2 diabetes and autosomal polycystic kidney disease, status post renal transplant 14 years prior. He takes tacrolimus and prednisone for immunosuppression. He lives in the rural Northeast, works on a farm, and killed a bat 1 month prior to admission.

\section{Questions for consideration:}

1. Where would you localize his deficits?

2. What is your differential based on this localization?

\section{GO TO SECTION 2}




\section{Section 2}

The constellation of multiple cranial nerve palsies and quadriparesis without cognitive deficits or sensory loss localizes to the brainstem (with or without contiguous spinal cord involvement), CSF space, motor nerves, neuromuscular junction (NMJ), or muscles. Downbeat nystagmus localizes to the cerebellar flocculus and surrounding brainstem areas and confirms some degree of parenchymal involvement. The depressed reflexes make an NMJ disorder and myopathy less likely. Although hyporeflexia and decreased tone are indicative of a lower motor neuron process, they can occur acutely with upper motor neuron damage. Overall, the findings localize to the brainstem, with potential extension into the CSF space, and are concerning for a brainstem encephalitis.
The differential diagnosis for brainstem encephalitis includes infectious, autoimmune, neoplastic, and paraneoplastic etiologies (table). Though the terms brainstem encephalitis and rhomboencephalitis are often used interchangeably and have similar etiologies, the latter refers specifically to inflammation restricted to the rhombencephalon (medulla, pons, or cerebellum). The most common causative infections in these syndromes include Listeria monocytogenes, enterovirus 71, and herpes simplex virus (HSV 1/2). ${ }^{1}$ Other infectious etiologies include bacteria, such as Mycobacterium tuberculosis, Borrelia burgdorferi, Treponema pallidum, Brucella, Salmonella, Nocardia, and Legionella. Viruses, chiefly enteroviruses, such as bulbar poliomyelitis, coxsackievirus A16, and echovirus; herpesviruses, such as human herpesvirus 6 (HHV-6), Epstein-Barr virus (EBV), cytomegalovirus (CMV), and varicella zoster virus (VZV); flaviviruses,

Table Differential diagnosis for brainstem encephalitis

Infections

Viruses

Enteroviruses: enterovirus 71, bulbar poliomyelitis, coxsackievirus A16, echovirus

Herpesviruses: herpes simplex virus 1 and 2, human herpesvirus 6, Epstein-Barr virus, cytomegalovirus, varicella zoster virus

Flaviviruses: West Nile virus, Japanese encephalitis virus, St. Louis encephalitis virus, Powassan virus

Other: HIV, rabies virus, John Cunningham virus

Bacteria

Listeria monocytogenes

Mycobacterium tuberculosis

Borrelia burgdorferi

Treponema pallidum

Brucella

Salmonella

Nocardia

Legionella

Fungi/parasites

Cryptococcus neoformans, toxoplasma gondii

Autoimmune syndromes

Guillain-Barre syndrome variants, Miller Fisher syndrome, Bickerstaff encephalitis, neuromyelitis optica

Behçet disease, systemic lupus erythematosus

Sjögren syndrome, sarcoidosis

Paraneoplastic syndromes

Anti-Hu and anti-Ma2 antibody-associated encephalitis

Neoplasia

Lymphoma, leptomeningeal metastases

Other

CNS post-transplant lymphoproliferative disease 
such as West Nile virus (WNV), Japanese encephalitis virus, and St. Louis encephalitis virus; HIV; and rabies virus can also cause brainstem encephalitis. ${ }^{1}$ Of note, WNV encephalitis incidence peaks in the summertime and can lead to flaccid paralysis and respiratory failure, especially in immunocompromised patients. Due to our patient's history of immunosuppression, other potential causes include toxoplasmosis, Cryptococcus, and progressive multifocal leukoencephalopathy.

The presence of rapidly progressive weakness with depressed reflexes also raises concern for Guillain-Barré syndrome (GBS). Miller Fisher syndrome (MFS) and Bickerstaff encephalitis (BE) are GBS variants that present with prominent eye movement abnormalities and are associated with antibodies against Gq1b gangliosides. Although MFS and BE classically present without overt limb weakness, significant clinical overlap exists with other GBS variants. ${ }^{2}$ Acute HIV seroconversion can also induce an inflammatory GBS variant with similar findings.
Noninfectious etiologies, such as sarcoidosis, Behçet disease, Sjögren syndrome, systemic lupus erythematosus, neuromyelitis optica, and lymphoma, and paraneoplastic syndromes, such as anti-Hu and anti-Ma2 antibody-associated encephalitides, can also cause a brainstem-predominant syndrome. ${ }^{1,3}$ However, the rapid symptom onset argues against these diseases. Botulism should be considered in cases of rapidly progressive bulbar and limb weakness, though patients classically present with descending paralysis and autonomic dysfunction, without fever.

CNS post-transplant lymphoproliferative disease (PTLD), thought to be due to reactivated EBV causing proliferation of host B lymphocytes, can also be considered given our patient's remote history of solid organ transplant. However, CNS PTLD occurs on average 4.5 years after organ transplant and rarely after 10 years. $^{4}$

\section{Questions for consideration:}

1. Would you begin empiric treatment? If so, for what?

2. What initial testing would you consider?

\section{GO TO SECTION 3}




\section{Section 3}

The patient was started on acyclovir, ceftriaxone, and ampicillin for empiric coverage of HSV, Lyme, and Listeria encephalitis, respectively.

Serum ganglioside antibodies, bacterial and fungal cultures, HIV, syphilis, and toxoplasma serologies were negative. Lumbar puncture revealed $85 \mathrm{mg} / \mathrm{dL}$ of protein, $54 \mathrm{mg} / \mathrm{dL}$ of glucose, and 28 leukocytes (76\% lymphocytes). CSF bacterial, fungal, and acid-fast bacilli cultures, paraneoplastic panel, cryptococcal antigen, HSV 1/2, VZV, Lyme, HHV-6, JC virus, enterovirus, EBV, and CMV PCRs, WNV, St. Louis encephalitis, California encephalitis, eastern and western equine encephalitis viral serologies (immunoglobulin $\mathrm{M}[\mathrm{IgM}] /$ immunoglobulin G $[\operatorname{IgG}])$, cytology, and flow cytometry were unrevealing. Rabies testing, including nuchal biopsy, serum and CSF IgM/IgG, and saliva PCR, was negative. MRI brain, cervical, thoracic, and lumbar spine showed leptomeningeal enhancement of the pons and cerebellum (figure). EMG and nerve conduction studies showed axonal polyneuropathy of indeterminate age, likely from the patient's diabetes. CT scans of the chest, abdomen, and pelvis (pan-CT) did not reveal a malignancy.

\section{Questions for consideration:}

1. Do these results change your differential?

2. Would you alter your treatment?

3. What further testing would you consider?

Figure MRI brain: Axial T2 fluid-attenuated inversion recovery (FLAIR), axial, and coronal T1 postcontrast sequences

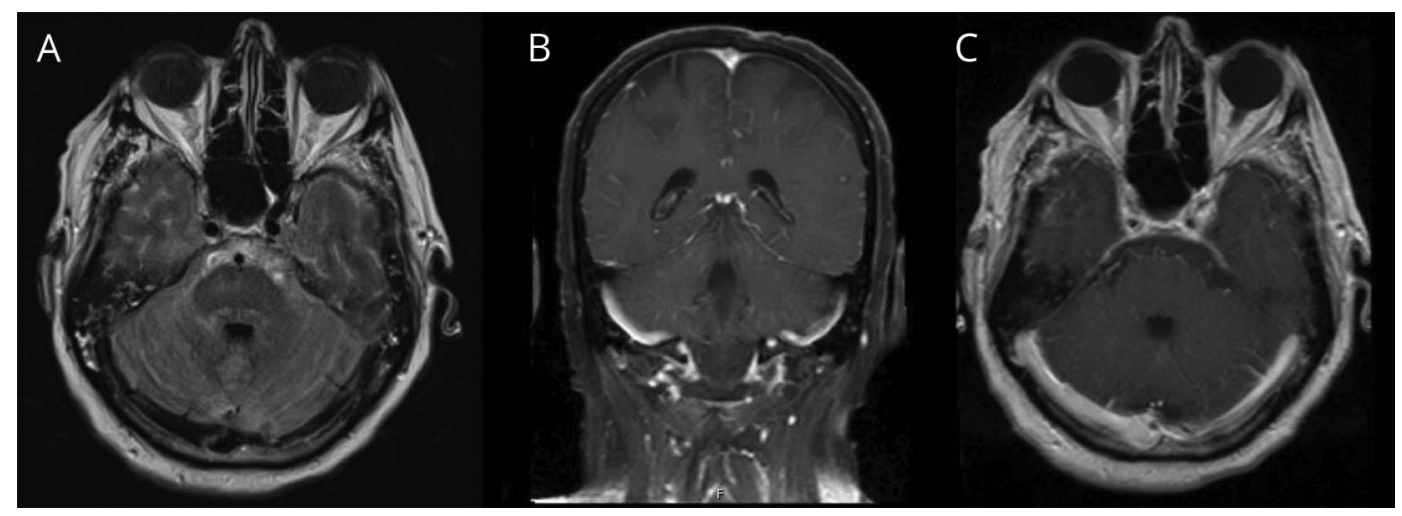

MRI brain shows T2 FLAIR signal change and leptomeningeal enhancement in the pons and cerebellum in (A) an axial T2 FLAIR sequence and in (B) coronal and (C) axial T1 postcontrast sequences. Of note, the sulcal T2 FLAIR signal in the temporal lobes (A) is thought to be secondary to hyperoxygenation, not inflammation. 


\section{Section 4}

The patient's MRI findings confirm a rhomboencephalitis. Although the rapid disease progression and presence of constitutional symptoms favor infection, the infectious workup so far has been unrevealing. Other less common infections should be considered; for example, Powassan virus (POWV), the tick vector of which is endemic to the rural Northeast, can cause a brainstem encephalitis and a clinical syndrome similar to that of WNV. The CSF lymphocyte pleocytosis and negative ganglioside panel argue against MFS and BE. The negative CSF paraneoplastic panel, pan-CT, cytology, and EBV PCR argue against a paraneoplastic syndrome, lymphoma, or PTLD.

The patient's antibiotics were narrowed to ampicillin to cover Listeria, and he completed a 21-day course given the low sensitivity of CSF culture for this organism. He was also given IV immunoglobulin to empirically treat autoimmune encephalitis.

The patient's CSF was sent to the Centers for Disease Control and prevention (CDC) for POWV testing. POWV CSF IgM serology returned positive 1 month into the patient's disease course, with high titers for the plaque reduction neutralization test.

The patient's strength gradually improved, though he required a tracheostomy and feeding tube. At discharge, he had normal cranial nerve function, $2 / 5$ strength in all extremities, and intact reflexes.

\section{Discussion}

POWV encephalitis is a tick-borne infection caused by a flavivirus, which is related to other arboviruses, including WNV, St. Louis encephalitis virus, and tick-borne encephalitis virus. POWV was first identified in 1958 in Ontario, Canada. From 2007 to 2016, the CDC reported 98 new cases across the United States, 88 of which were neuroinvasive, mostly occurring in Northeastern and Midwestern states in the spring through fall. ${ }^{5}$

In the Northeast, POWV is carried by the Ixodes scapularis tick, the same vector that transmits Lyme disease, babesiosis, and anaplasmosis. POWV infection typically presents with an initial febrile illness that can progress to encephalitis or encephalomyelitis after an incubation period of 1-5 weeks. ${ }^{6,7}$ Prior case reports have described both flaccid and spastic paralysis in adults with POWV. ${ }^{6-8}$ Around $10 \%$ of infections are fatal, with over $50 \%$ of patients having permanent neurologic deficits, including hemiplegia, ophthalmoplegia, headaches, and memory loss. ${ }^{6}$ CSF analysis typically shows a neutrophil or lymphocyte pleocytosis with elevated protein. MRI findings commonly demonstrate T2 hyperintensities (with or without enhancement) in the brainstem, cerebellum, deep gray structures, and cortex, though normal brain and spine imaging have been seen in some cases. ${ }^{7}$ Postmortem pathologic evaluation of patients with POWV infection has revealed neuronal destruction in multiple areas of the CNS, including the cortex, basal ganglia, thalami, brainstem, spinal cord, anterior horn cells, and cerebellum, which may explain the differing patterns of neurologic deficits that have been reported. ${ }^{6}$ It is reasonable to assume that our patient's age and history of immunosuppression made him more susceptible to profound disease sequelae, given that these 2 characteristics are associated with increased severity of other flaviviridae infections, such as WNV. ${ }^{9}$ The patient's CSF pleocytosis is not unexpected given his low dose of prednisone $(5 \mathrm{mg})$ and the presence of leptomeningeal enhancement, which indicates that he can generate at least a partial immune response.

The diagnosis of POWV is made by a compatible clinical syndrome combined with culture of the virus, detection of IgM antibodies by ELISA with a confirmatory plaque neutralization test, or a 4-fold rise in POWV antibody titers. ${ }^{10}$ There is no vaccine or disease-specific treatment for POWV infection, thus minimizing tick exposure is the best preventative measure. Treatment is largely supportive. While there have been reports of using high-dose corticosteroids and IV immunoglobulin in patients with POWV encephalitis, their effect on disease outcome is unclear. ${ }^{6}$

POWV infection has been increasingly documented over the last decade, whether due to augmented testing for the virus or true disease emergence. A high index of suspicion is needed to diagnose POWV encephalitis. Clinicians in the Midwest and Northeast should consider testing for this disease in patients who present with an acute flaccid paralysis in the spring through fall with risk factors for tick exposure.

\section{Author contributions}

Denise $\mathrm{Xu}$ : conception and drafting of the original manuscript, revisions, and figure creation. Kathleen Murphy: editing and critical revisions to the intellectual content. Ramani Balu: editing and critical revisions to the intellectual content. Jon Rosenberg: conception and drafting of the original manuscript with critical revisions.

\section{Study funding}

No targeted funding reported.

\section{Disclosure}

The authors report no disclosures relevant to the manuscript. Go to Neurology.org/ $\mathrm{N}$ for full disclosures.

\section{References}

1 Jubelt B, Mihai C, Li TM, Veerapaneni P. Rhombencephalitis/brainstem encephalitis. Curr Neurol Neurosci Rep 2011;11:543-552.

2 Wakerley BR, Uncini A, Yuki N. Guillain-Barré and Miller Fisher syndromes: new diagnostic classification. Nat Rev Neurol 2014;10:537-544.

3 Dalmau J, Rosenfeld MR. Paraneoplastic syndromes of the CNS. Lancet Neurol 2008; 7:327-340.

4. Lake W, Chang JE, Kennedy T, Morgan A, Salamat S, Baskaya M. A case series of primary central nervous system post-transplant lymphoproliferative disorder: imaging and clinical characteristics. Neurosurgery 2013;72:960-970. 
5 Powassan virus disease cases reported to CDC by state of residence, 2007-2016. In: CDC Powassan Virus Home [online]. Available at: cdc.gov/powassan/statistics.html. Accessed December 26, 2017.

6. Hermance ME, Thangamani S. Powassan virus: an emerging arbovirus of public health concern in North America. Vector Borne Zoonotic Dis 2017;17: 453-462.

7. Piantadosi A, Rubin DB, McQuillen DP, et al. Emerging cases of Powassan virus encephalitis in New England: clinical presentation, imaging, and review of the literature. Clin Infect Dis 2016;62:707-713.
8 Birge J, Sonnesyn S. Powassan virus encephalitis, Minnesota, USA. Emerg Infect Dis 2012;18:1669-1671.

9. Ravindra KV, Freifeld AG, Kalil AC, et al. West Nile virus-associated encephalitis in recipients of renal and pancreas transplants: case series and literature review. Clin Infect Dis 2004;38:1257-1260.

10 Arboviral diseases, neuroinvasive and non-neuroinvasive, 2015 case definition. In: CDC NNDSS [online]. Available at: cdc.gov/nndss/conditions/arboviral-diseasesneuroinvasive-and-non-neuroinvasive/case-definition/2015/. Accessed December 26, 2017. 


\section{Neurology}

Clinical Reasoning: A man with rapidly progressive weakness and respiratory failure

Denise Xu, Kathleen Murphy, Ramani Balu, et al.

Neurology 2018;91;e686-e691

DOI 10.1212/WNL.0000000000006003

This information is current as of August 13, 2018

Updated Information \&

Services

References

Subspecialty Collections

Permissions \& Licensing

Reprints including high resolution figures, can be found at:

http://n.neurology.org/content/91/7/e686.full

This article cites 8 articles, 0 of which you can access for free at: http://n.neurology.org/content/91/7/e686.full\#ref-list-1

This article, along with others on similar topics, appears in the following collection(s):

Clinical neurology examination

http://n.neurology.org/cgi/collection/clinical_neurology_examination Encephalitis

http://n.neurology.org/cgi/collection/encephalitis

Viral infections

http://n.neurology.org/cgi/collection/viral_infections

Information about reproducing this article in parts (figures,tables) or in its entirety can be found online at:

http://www.neurology.org/about/about_the_journal\#permissions

Information about ordering reprints can be found online:

http://n.neurology.org/subscribers/advertise

Neurology ${ }^{\circledR}$ is the official journal of the American Academy of Neurology. Published continuously since 1951, it is now a weekly with 48 issues per year. Copyright @ 2018 American Academy of Neurology. All rights reserved. Print ISSN: 0028-3878. Online ISSN: 1526-632X.

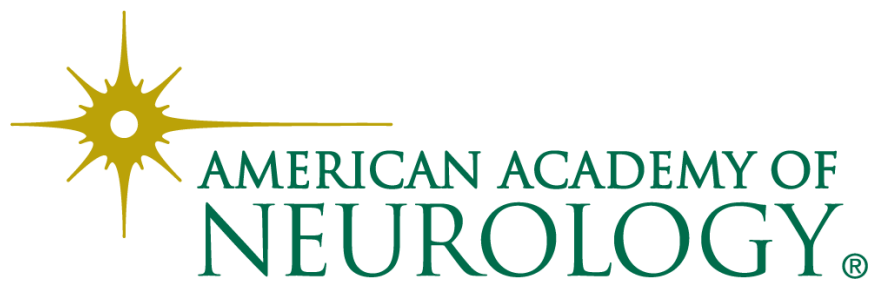

